\title{
A Comparison of Dissolution Results Using 0-ring vs. Clipped Basket Shafts
}

Vivian Gray,* Mary Beggy, Robert Brockson, Nancy Corrigan and John Mullen

Bristol-Myers Squibb Company

Wilmington, Delaware
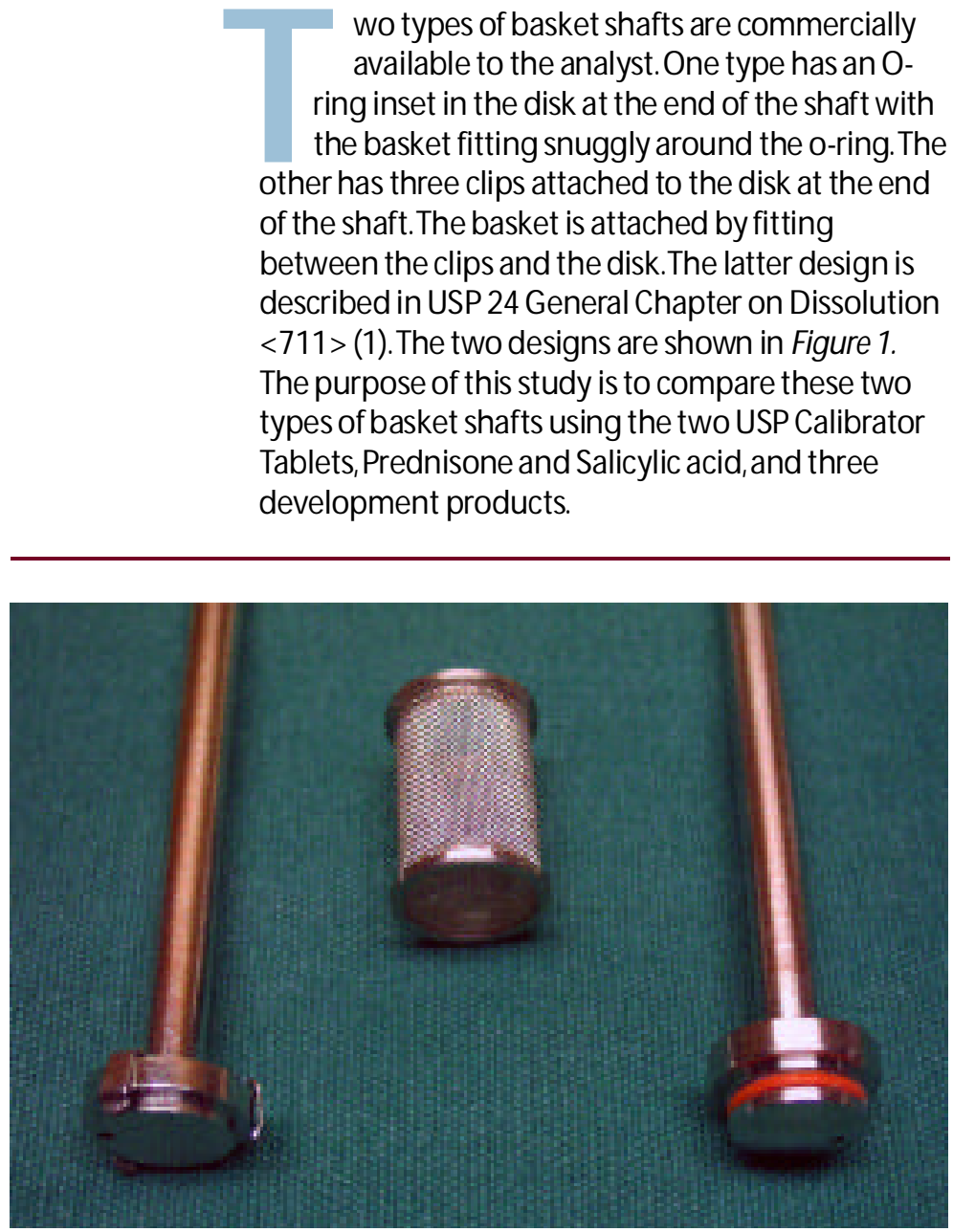

Figure 1 email: vivian.a.gray@dupontpharma.com *to whom correspondence should be addressed

\section{Compound A}

Tablet-erosion type

Apparatus,speed:1 (basket), $50 \mathrm{rpm}$

Medium, volume: $0.05 \mathrm{M}$ sodium carbonate

containing $0.25 \%$ sodium lauryl sulfate adjusted

to $\mathrm{pH} 9.5,900 \mathrm{~mL}$

Time points- $10,20,30,45$ minutes

UV analysis@265 nm

\section{Compound B}

Tablet--disintegrating

Apparatus,speed:1 (basket),50 rpm

Medium, volume: $0.01 \mathrm{~N}$ hydrochloric acid,

$900 \mathrm{~mL}$

Time points - 10,20,30,45,60 minutes

UV analysis @305 nm

\section{Compound C}

Capsule

Apparatus, speed: 1 (basket), 100 rpm

Medium, volume: $0.5 \%$ sodium lauryl sulfate in

$0.001 \mathrm{~N}$ hydrochloric acid

Time points- $10,20,30,45$ minutes

HPLC-Symmetry C18 (5 $\mu$ ) 3.9x150 mm

Column temperature $40^{\circ} \mathrm{C}$

Flow rate $\quad 1 \mathrm{~mL} / \mathrm{min}$

Wavelength $\quad 220 \mathrm{~nm}$

Injection volume $25 \mathrm{uL}$

Mobile phase- 50/50 acetonitrile/water with $0.1 \%$ TFA

\section{USP Prednisone Calibrator Tablet}

Disintegrating tablet

Apparatus,speed:1 (basket), 100 rpm

Medium, volume:water, $900 \mathrm{~mL}$

Time point-30 minutes

UV analysis@242 nm

One run was performed on the Prednisone calibrator tablet and three products. For each run the tester was equip ped with three o-ring and three clipped basket shafts. For the Salicylic Acid Tablets, two runs were performed in tandem, the first with the 0 -ring shafts and the second with clipped shafts. For each basket shaft type there were three replicates $(n=3)$, except Salicylic Acid which was $n=6$. The basket test methods used are as follows:

\section{USP Salicylic Acid Calibrator Tablet}

Non-disintegrating Tablet

Apparatus, speed:1 (basket), $100 \mathrm{rpm}$

Medium, volume:0.05M phosphate buffer

adjusted to $\mathrm{pH} 7.4,900 \mathrm{~mL}$

Timepoint-30 minutes

UV analysis@296nm 


\section{Results}

The data for the calibrator tablets in Tables 1 and 2 are a comparison at the 30 -minute time point. Using the Student ttest, $p=0.0147$ for Prednisone Tablets and $p=0.0079$ for Salicylic Acid Tablets. Both are statistically different, however the USP Salicylic Acid Tablets data are so tight that the difference may not be relevant. The USP Prednisone Tablets dissolution results showed the most dramatic difference, with results being higher with the clipped basket shafts. The graphical comparisons using the development products are shown in Figures 2,3, and 4. Each product gave very similar profiles for each basket shaft type.

\section{Conclusions}

There was no difference between the two basket shaft types for the three development products and USP Salicylic Acid Tablets. However, the USP Prednisone CalibratorTablets did show a significantly different dissolution rate. The difference showed a higher dissolution rate using the clipped basket shaft design. The clipped basket shaft is the official USP design, however there are some drawbacks to this design. The clips protrude and disturb the fluid flow in the vessel.The clips can weaken over time and cause the basket to be attached too loosely to the shaft-increasing the chance for wobble. When using robotic dissolution testers, a robotic arm can remove the 0 -ring type basket more efficiently.

Since the 0-ring style is not an official design, the analyst should show that it does not give results different from the clipped shafts when testing their products. As part of valida-

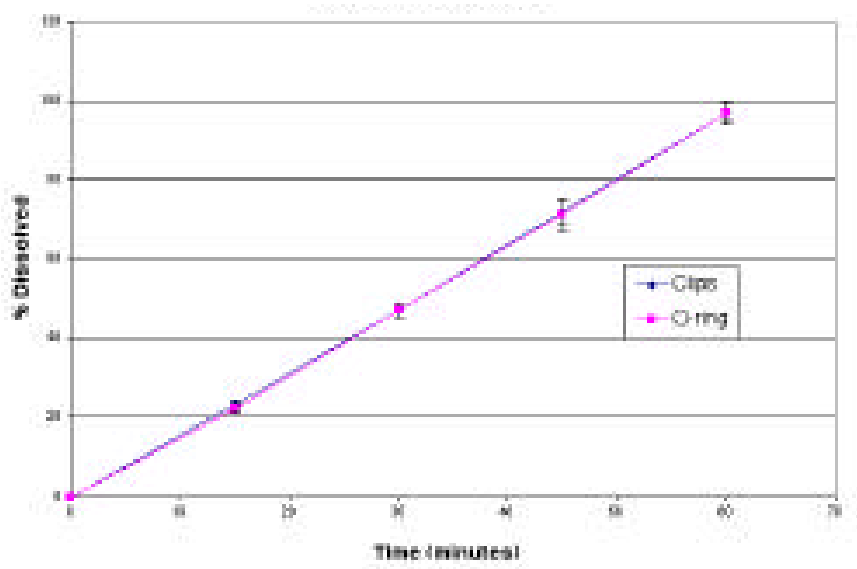

Figure 2. Compound A:Tablet - Erosion Type
Table 1. Comparison of Dissolution Resultsfor USP PrednisoneCalibrator Tablets, Percent Dissolved in 30 Minutes

\begin{tabular}{|lll|}
\hline Basket attachment type & 0-ring & Clips \\
\hline \% Dissolved & 78 & 82 \\
\hline & 79 & 81 \\
\hline Mean & 77 & 84 \\
\hline SD & 78 & 82 \\
\hline
\end{tabular}

Table 2. Comparison of Dissolution Resultsfor USP Salicylic Acid Calibrator Tablets,Percent Dissolved in 30 Minutes

\begin{tabular}{|lll|}
\hline Basket attachment type & 0-ring & Clips \\
\hline \% Dissolved & 25 & 27 \\
\hline & 26 & 26 \\
\hline & 26 & 27 \\
\hline & 26 & 27 \\
\hline & 25 & 27 \\
\hline Mean & 26 & 29 \\
\hline SD & 26 & 27 \\
\hline
\end{tabular}

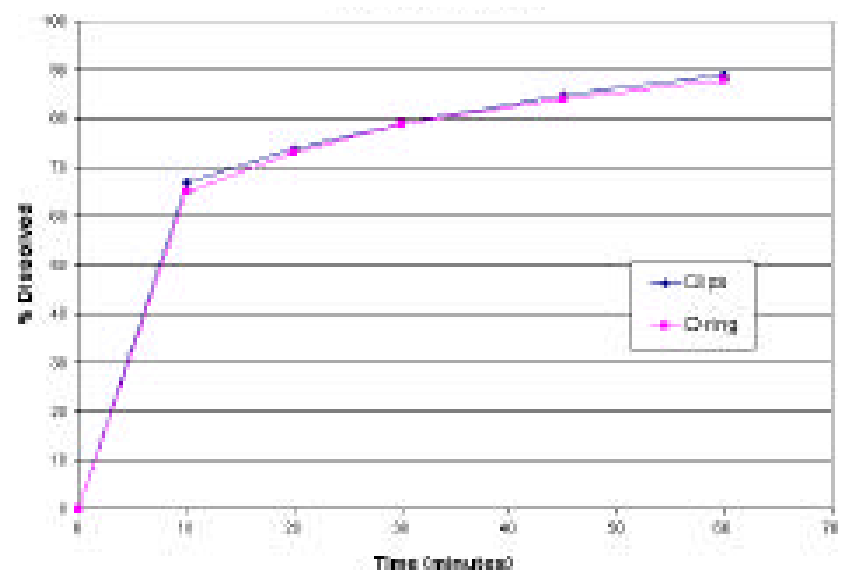

Figure 3. Compound B: Tablet Disintegrating Type 


\section{O-ring vs. Clipped Basket ... continued}

tion, the two basket shaft types should be compared and equivalence shown.If the types do not give comparable results, there could be problems with technology transfer. In addition, if a regulatory agency performs the dissolution test on a product using the USP procedure, the results obtained could be different.

\section{References}

(1) USP 24/NF 19, National Publishing Company, Philadelphia,(1999), page 1942.

\section{Acknowledgments}

Brenda Rice, Bristol-Myers Squibb Company David Lloyd, Bristol-Myers Squibb Company Jay Blazer, Bristol-Myers Squibb Company

Address correspondence to:

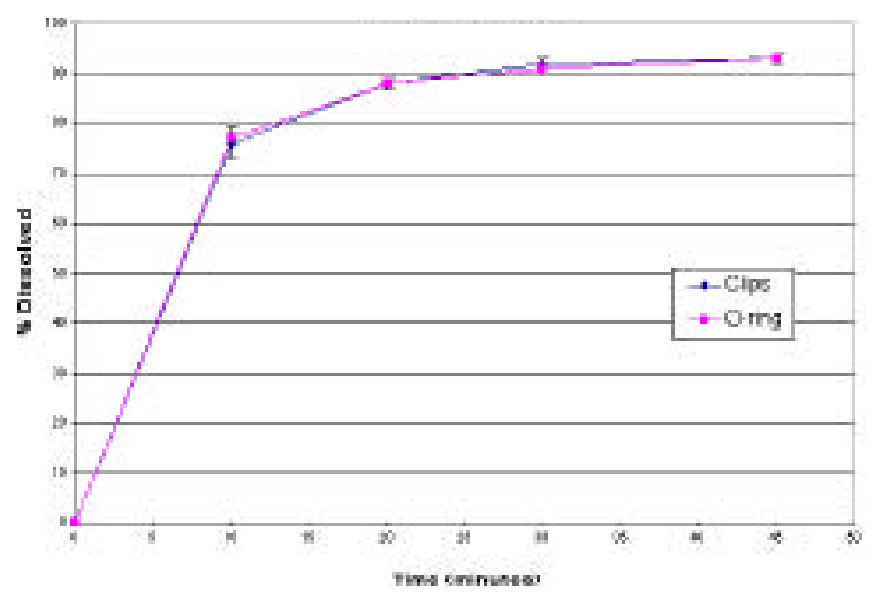

Vivian A. Gray

Bristol Myers Squibb Company

Experimental Stationn,E353/216A

Wilmington,DE 19880-0353 